This is a pre-publication draft of Leaver, T. (2019). Co-creating Birth and Death on Social Media. In Z. Papacharissi (Ed.), A Networked Self: Birth, Life, Death. London and New York: Routledge, pp. 35-49.

\title{
Co-Creating Birth and Death on Social Media
}

Tama Leaver, Curtin University, t.leaver@curtin.edu.au

\section{Introduction}

While social media is, by definition, about connecting multiple people, many discussions about social media platforms and practices presume that accounts and profiles are managed by individual users with the agency to make fully-informed choices about their activities. When discussing children, especially younger children, their agency is at times characterised as partial, or emerging, but with the presumption that with sufficient time they will eventually reach the same (presumed) status and ability as adult users (Livingstone \& Third, 2017). At the other end of life, at the moment of death, the social media traces and online presences that persist after a user has passed away also present challenges in terms of agency. While there is an increasing push to include some sort of instructions about digital property in wills, these instructions are currently few and far between. Some platforms have deployed algorithmic solutions which have begun to address the reality of deceased users, but these are, at best, partial and largely insufficient responses. With these two figures in mind, I argue that the very young-from conception to birth and early infancy-and the recently deceased both act as liminal figures where the question of their (lack of) agency on social media highlights some of the ongoing challenges in presuming that social media traces can always be the responsibility of users with full, or even partial, agency. Rather, using a range of examples, I argue in this chapter that more encompassing ways of thinking about the relationship between social media, networked selves and identities, are needed. Drawing on work from the creative industries, I suggest that the term co-creation can be reframed to emphasise the way that social media almost always entails creating other people's identities as much as our own. Parents and carers are the first arbiters and co-creators of a young person's life, making a large number of important choices about what sort of private or public online presence a newly born baby will have, how that presence will develop over time, on which platforms, and under which circumstances. Parents, in effect, can choose to name their children into being online, and in doing so must navigate the parental joys of sharing whilst balancing this against the rights of the child to, amongst other things, privacy in the present and future. At the other end of life, but in functionally similar ways, the loved ones left behind by the recently deceased will often need to make decisions about which social media profiles and traces persist after that user has died, how these traces will be (re)framed, and what online spaces will persist (if any), possibly in the form of online memorials. Moreover, both ends of life are now situated in an online context where real identities and real names, which persist over time, are both expected and demanded by the policies and practices of online platforms. The use of real names on social media amplifies the impact and longevity of social media traces, whether early or late in life. In outlining the challenges inherent in framing the very young, and the recently deceased, online, I argue in this chapter that a broader sense of agency and impact is needed across all life-stages on social media. A wider lens in terms of the way users contribute to the stories of each other on social media may well assist us all in making decisions about online material that inevitably impact the lives and legacies of other people. 


\section{From Networked to Co-Created Selves}

Since the inception of the web, and the internet before it, questions about identity online, especially questions of authenticity and selfhood, have proven particularly complex. The work of sociologist Erving Goffman (1959) has been particularly influential, utilised by many scholars exploring the way that performances of self differ according to the (imagined) cohort and context in which they are seen (Pearson, 2009). One of the key challenges, though, for online identities and authenticity is problem of context collapse insomuch as this concept highlights the fact that identity traces and communication may (re)appear in all sorts of different contexts, often quite different from the initial one for which a particular performance was shaped (Davis \& Jurgenson, 2014; A. E. Marwick \& boyd, 2011). A popular framework for mapping the complexities of identities and selves is the broad notion of the networked self (Papacharissi, 2010). Focusing on the circulation of networked selves within networked publics, danah boyd (2010) posits some key affordances that describe the way social media traces circulate:

- persistence - once created, generally social media traces do not disappear in part because maintaining and analyzing these traces drive the core business model of many online platforms;

- replicability - once identity information or material is created and shared online, in whatever form, it takes trivial effort to create copies, often stored in different contexts, governed by multiple sets of rules and conditions; and

- scalability - the difference between making one copy, or a million copies, is trivial, so the potential to shift contexts, and even be greatly amplified (or 'go viral'), is inherent in most social media material.

Additionally, and quite importantly, Patricia Aufderheide (2010) adds that networked selves and online identities largely circulate on platforms which are corporately owned, subject to their own terms of use rather than those of a specific national government. In many instances where control of an individual's online identity is insufficient or incomplete, there is a call for better privacy controls and better tools to allow individuals to shape the audiences that can see their information (boyd, 2008). These calls for more agency are important, but further thought is required in contexts where the identity or digital traces in question relate to people who cannot exercise this agency; babies and the recently deceased are two such groups (Leaver, 2015b).

While it was once relatively easy to have multiple identities, use pseudonyms, and delete histories relating to one username or another (van der Nagel, 2017), the rise of large corporate platforms which encompass multiple services, apps and functions has seen an increasing push toward persistent singular identities online. The so-called real name web is often championed in terms of providing more security or safety online, but a more cynical interpretation notes that a single 'real' identity makes the business model of Facebook or Google - selling targeted advertising based on deep personal profiles - far more effective (van Dijck, 2013; van Zoonen, 2013). José van Dijck (2014) argues further than the linking of real names to a long digital history including every piece of content shared by and about that person, as well as every communicative utterance they make online, constitutes dataveillance, a new, persistent digital form of surveillance. In this platform-driven context, when parents post information about their babies and children online, a real-name web and the persistence of digital traces mean that when they are older 
these young people may confront a networked self with their name, but which has been created without their agency or, initially, their choice (Leaver, 2015a). Similarly, after a user dies, their social media legacy may persist, but clearly how these traces are managed must rely on other people and platforms to be shaped, stored, mourned, memorialized or entirely deleted (Leaver, 2013).

While models such as the networked self always imply the importance of platforms and other people (constituting the network) in terms of identities online, this chapter argues that more explicit models and terminology are needed to highlight just how vital people are in creating, shaping and maintaining each other's identities, selves and legacies online. Within the creative industries, the term 'co-creation' initially signaled more meaningful and collegial creation of content across multiple forms and producers, rather than a single authorial or directorial voice (Jenkins, 2006). Co-creation as a model was then further developed to describe increasing user input into the design of videogames and other large-scale media productions (Banks \& Humphreys 2008; Banks and Potts 2010). Banks and Burgess (2010, p. 298) describe co-creation as:

a purely descriptive term that highlights the ways that users or consumers, within the constraints and affordances of platforms provided by others, collectively contribute to the social, cultural and economic value of the media products and experiences associated with those platforms; and likewise, it indicates the ways in which platform providers (however imperfectly) integrate user-participation into their own models of production.

As recent work from Hutchinson (2017) argues further, as a concept co-creation has subsequently been deployed in a range of different commercial, creative and conceptual contexts, although always marking the deepening relationships between the roughly bifurcated producerly side (media creators, platforms, companies) and the user side (user created content makers, users, players). Part of my argument in this chapter is that the term co-creation can be borrowed from these initial contexts and refocused to explicitly highlight the way users co-create each other on social media. In part, co-creation can make visible the 'social media contradiction' which Leaver and Lloyd (2015, p. 162) describe thus:

a social media contradiction may arise where users focus on the social elements - often acts of communication and sharing which are thought of as ephemeral and in the moment, comparable to a telephone conversation - while the companies and corporations creating these apps are more focused on the media elements, which are measurable, aggregatable, can be algorithmically analysed in a variety of potentially valuable ways, and can last indefinitely.

Co-creation as a term and concept can be useful in making visible this contradiction and making it explicit that all use of social media is the creation of media. Our identities, our children's identities and even the web presences of deceased users are all amalgams of online media. Every comment, message, photo, video, like, retweet, or post is media production; conceptualizing this as co-creation can make visible that all communicative acts in digital forms are always simultaneously media production. Moreover, users are frequently producing the media that constitutes other people's online selves, identities and presences, not just their own.

Borrowing co-creation as a concept from work in the creative industries is also useful as the initial work always kept in mind the uneven terrain in terms of platforms and users. Co-creation takes place in quite bounded contexts, and those boundaries are often points of contention. Platforms are never considered neutral; as Hutchinson (2017, p. 19) argues "the platforms are actively participating in the act of content 
creation too". Most importantly, then, in harnessing co-creation to illuminate the ways users create each other's online identities, the concept emphasizes: multiple ways users can and do contribute to other users identities; the unequal relationship between users and the platforms on which they often create and curate digital identity traces; and that collaboration is always a process where negotiation is generally needed to ensure that creation benefits both the user creating and the individuals whose identity is formed, in part, by that creation. This is evident on most social media platforms, as users are always contributing to the online presences of each other, whether that's in a visible form, such as sharing and tagging photos of one another, or in less obvious ways, such as simple conversations and messages between individuals which are nevertheless algorithmically analysed by platforms to determine both advertising and media or news feeds. Yet these are contexts where users generally have agency and can dialogue back, often deleting, removing or untagging material that does not fit into their own personal identity curation online. For the remainder of this chapter, however, I want to emphasise those cocreative moments where the person or groups being named, described and curated do not have the agency to speak back, to immediately be involved in discussing or debating how they are (re)presented online. In explicating how identity co-creation operates in terms of babies and the recently deceased, my aim is to illuminate the power and responsibility users have in shaping each other's identities, both at these more visible moments, but also in terms of social media use more generally.

\section{Ultrasounds: Presence Before Birth}

The sharing of sonograms, the images created during fetal ultrasound scans, has become a rite of passage for pregnant mothers across the world. In last 15 years, that ritual has increasingly taken place online, with Facebook or Instagram posts showing the usual 12 or 20-week ultrasound images becoming one of the most normal ways of announcing a pregnancy socially in the Western world (Lupton, 2013). Along with the ever-expanding range of pregnancy-related apps, the sharing of ultrasounds on social media tends to exacerbate existing social and medical practices, including giving the fetus a voice as if it is 'already a communicative person in its own right' (Lupton \& Thomas, 2015) as well as the tendency to visualize only the fetus itself, occluding the maternal body and mother altogether. In a study looking at 3 months of images publicly shared using the \#ultrasound hashtag on Instagram in 2014, Leaver and Highfield (2018) found a range of practices, including mothers taking selfies on the way to and from ultrasound scans, as well as photos of parents holding physical ultrasound photos in front of them, both of which bring the mother's body and agency back into focus. Significantly, when thinking about cocreation, $34 \%$ of the fetal sonograms qualitatively coded still included personally-identifiable metadata, including the mother's name, the facility where the scan was taking place, the mother's date of birth, and the expected due date of the fetus. While this metadata is often text inside the image (not separately entered as text in the image's caption), it is nevertheless easily machine-readable. As Facebook (Instagram's parent company and owner) scrutinizes every available piece of personal information, it is reasonable to presume that Facebook utilizes these ultrasounds both to inform the advertising visible to pregnant parents, but also to extrapolate the beginnings of a new profile, visible to the company, based on the visualized fetus who has not yet even been born. As a further indicator of the social norms of sharing ultrasounds, the biggest ultrasound machine manufacturers today include systems such as Tricefy 
which not only store all of a customer's ultrasound images on the cloud for later access, but this access comes with built-in buttons for sharing these images directly via a user's social media platforms.

A very similar example is the sharing of prenatal images announcing the gender of an unborn child. Gender-reveal parties, where expectant parents unveil to an assembled group of friends and family some sort of object - often pink or blue balloons, or the inside colour of a cake - immediately marking the unborn child's body and positioning social expectations by categorizing them as either 'It's a Boy' or 'It's a Girl' (with absolutely no acknowledged grey areas in between). While intimately and affectively tied to the experience of pregnancy and preparing for a new child, the recent popularization of gender reveal parties, and the sharing of these on social media, has implications both for identity formation and the child's freedom of expression and privacy. As Gieseler (2017, p. 10) argues:

The ritualized performance of the gender-reveal creates a space for parents to articulate and mark the identities of their unborn children. [These parties replicate] what adults have learned

from their own gendered construction, placed upon the intimate, private, blank canvas of the foetus and propelled into the digital, social, public world.

As of November 2017, there were, for example, over 450,000 public images on Instagram using the \#genderreveal hashtag and a cursory examination confirms that the vast majority do depict gender reveal parties. While the focus of this chapter is on social media, gender reveal parties also remind us that parents and others co-create their children's identities in a range of other ways and contexts too, whether shared on social media or not. Yet the influence of gender reveal parties, and the sharing of these on social media, clearly contributes to the initial online identity of the unborn child, an identity crafted (at times, probably unwittingly) by their expectant parents.

The examples of sharing ultrasounds and gender reveal parties on social media demonstrate the ways that parents, families and loved ones begin to co-create the identities of young people online, even before those people are born. In the context of a real name web with persistent identities, these initial online footprints may well be part of that young person's networked self for their entire lives. Beyond the simple act of creating their children's initial online presence, these co-creative acts must also be framed in terms of children's rights. As Sonia Livingstone and Amanda Third (2017, p. 662) have argued, 'children's rights are the responsibility of parents and care-givers', including the right to privacy, a right which the UN Convention on the Rights of the Child states begins at birth. Positioning the sharing of social media material about the unborn as co-creation more explicitly asks parents and others to consider the lasting online legacy they are leaving for their children to inherent, and whether this legacy is consistent with young people's right to privacy.

\section{Infant Wearables and Intimate Surveillance}

As Sophia Johnson (2014, p. 346) has argued, pregnancy apps and domestic monitoring devices are part of a wider "device-ification'\|of mothering" which emphasizes the regulation, normalisation and datafication of pregnancy, implicitly devaluing subjective, embodied maternal experiences. After a baby is born, these trends continue with both baby monitoring apps, social media commentary on shared baby images, and the relatively new area of infant wearables. Infant wearables are monitoring devices which are worn on an infant's body to quantify specific biological activities and states which are then wirelessly transmitted as data to central server before being interpreted and displayed to parents, usually via a mobile app (Leaver, 2017). One of the most well-known infant wearables is the Owlet 'Smart Sock' which 
infants wear while sleeping; the device records the infant's biometric data, including blood-oxygen levels, heart-rate and movement, and transmits these via a base station to the cloud. Once interpreted, the information is then relayed to smartphone or tablet apps which display vital signs and a series of traffic light indicators (green all is okay, yellow action is needed, red immediate intervention needed). The Owlet company carefully market their device as providing weary parents with the 'peace of mind' of knowing that their infants are sleeping healthily in another room. While many parents appear to believe the Owlet can prevent Sudden Infant Death Syndrome (SIDS), it is important to note that it is not a medical device, nor medically certified, and is in fact being the subject of warnings from the American Paediatrics Association (Bonafide, Jamison, \& Foglia, 2017; King, 2014), who argue the device can provide both false warnings and false reassurance, increasing both risk and anxiety for new parents. However, with a welloiled advertising machine and ringing endorsements from celebrity parents such as Katherine Heigl (2017), the Owlet and a range of related devices continue to sell well across the US, Australia and elsewhere.

The Owlet and infant wearables typify what I have defined elsewhere as intimate surveillance:

the purposeful and routinely well-intentioned surveillance of young people by parents, guardians, friends, and so forth. The surveyed have little or no agency to resist. On one level, intimate surveillance points to the limits of most surveillance models, in that they are incomplete in trying to address subjects who have no agency or awareness of the means of resistance (for obvious reasons) (Leaver, 2015a, p. 153).

The term intimate surveillance is intended to emphasise that the monitoring undertaken by parents and loved ones is motivated by care. Apps and devices are purchased to try and provide the very best for their children, and labelling this surveillance challenges the unproblematic sales pitch of these devices just providing a benevolent peace of mind. Indeed, the Owlet and other infant wearables literalize the ultimate business model of social media; they record, encode and digitize every movement, motion and signal an infant's body makes, capturing this as a form of big data which can then be harnessed and monetized in various ways. The Owlet also typifies the intersection of social media apps with medical and health platforms, along with a related business model which involves the sales of both the apps or devices themselves but also, and often more importantly, the second level where the data generated by these devices is collected, aggregated, stored and analysed as a form of big data which can be monetised in various ways (van Dijck \& Poell, 2016). The Owlet company have been very explicit in their corporate promotional material that they see the most significant value in their big data set which they claim is the "the largest data set about infant health and sleep and wellness and safety that's ever been collected" (Owlet Baby Care, 2015).

Rather than accepting at face value that these devices are just providing 'peace of mind', situating the creation of big data about infants as a form of co-creation begs parents and others to ask better questions about how their children's data will be used. Who will own the data? In what circumstances can it be shared? Is it stored anonymously, and even if it is, how hard is it to use a unique dataset of biometric data to reassociate 'anonymous' data with a particular subject? Many Owlet users no doubt ask these questions already, perhaps being surprised to find the company claim complete ownership of all data recorded, but the lens of co-creation with its implied shared responsibility can make the urgency of asking these questions more evident. Similarly, when companies like Owlet turn around and offer to sell users back their own children's data in a new app with new costs, as is the case with their new Connected Care 
app (Owlet Baby Care, 2017), it makes visible the core of their business model. Balancing the seeming reassurance these devices offer with the long-term privacy of children's personal data is conceptually difficult, trying to envisage what benefits and what harm might result. Could, for example, an insurance company one day buy-out the Owlet business and utilise the historical health data to calibrate the costs of future health insurance? The only certainty with this form of big data is that it will be used in ways not immediately clear on the day that the data is captured. Moreover, the design of these devices which encourage intimate surveillance can displace other narratives of care, and encourage parents to continue digital surveillance of their children in other forms as they grow (Rettberg, 2014). Situating the generation of children's big data as part of co-creation might allow parents to better imagine the choices they are making when using these devices, and the possible ramifications for their children's right and privacy in the future.

\section{Digital Death on Facebook and Google}

While questions of agency and co-creativity might seem irrelevant when considering digital death and deceased users, the complexities in dealing with posthumous digital material in almost any form make the question of shared responsibility even more pressing. There are many boundaries, including copyright, contracts, and Terms of Use, which make the process of preserving or inheriting digital media content challenging, with unclear rules and norms which differ by nation, and even by state within specific nations (Bellamy, Arnold, Gibbs, Nansen, \& Kohn, 2013). Beyond the many challenges of preserving the integrity of social media traces that existed on dynamic platforms (Acker \& Brubaker, 2014), most social media platforms do not have explicit policies to deal with deceased users until their presence becomes publicly problematic.

For Facebook, deceased users became particularly problematic when they first introduced the friend suggestions algorithm which used overlapping friend networks to suggest new users to each other that had network connections but were not yet Facebook friends. As soon as this feature was turned on, people started reporting the distressing scenario of receiving friend suggestions for people they knew to be deceased. At that time Facebook did not know how to deal with deceased users, but it was clear that foisting the recently deceased as friend suggestions was an uncomfortable experience for many users. Facebook's eventual response was to invent memorialized pages; when Facebook is notified or becomes aware that a user has died, their default solution is to lock the page into a memorialized state which respects the profile's final privacy settings, which usually means it allows existing friends to post on the page, to leave photos and memories as a memorial, but no further changes are possible (Leaver, 2013; A. Marwick \& Ellison, 2012). While memorialized pages showed Facebook's good-faith attempt to address the deceased suggested friends problem, memorialization is a still a very blunt process; if the final posts on a deceased users page were insults or poor attempts at humour, memorialization locks these in place as the user's final words, with no recourse to edit them. More recently, in an attempt to provide some nuance, Facebook introduced the Legacy Contact function (Callison-Burch, Probst, \& Govea, 2015) which allows Facebook users to nominate a Legacy contact who will be given the ability to write a final post, change the cover photo and manage new friend requests after a user passes away. The Legacy Contact thus gives family and loved ones some recourse to edit and curate a recently deceased person's account, although still with many restrictions, and the Legacy contact does not have access to other data, such as the content of Facebook messages. While Facebook's Legacy function was designed with the best of 
intentions (Brubaker \& Callison-Burch, 2016), it is a partial, automated and algorithmic solution which relies on a uniformity of experience as well as social media users having sufficient foresight and planning to actually nominate a legacy contact. Given many people die without leaving a will of any form, the odds of even the majority of Facebook users knowing about and using the Legacy Contact function are minimal at best.

Like Facebook, Google initially had no ready response to the question of data left by deceased users, and languished behind an incredibly complex legal process until 2013 which made the only way to access a deceased user's material being via an official court order. However, in 2013 Google launched their software solution to digital death: the Google Inactive Account Manager (Google, 2013; Lardinois, 2013). The Inactive Account Manager allows any Google account holder to nominate one or more people who should receive copies of data from certain tools, such as YouTube, Gmail or Google Drive; or to nominate data from specific tools that should be completely deleted upon the user's death (or, in software terms, the designated period of inactivity). While the Inactive Account Manager is definitely better than nothing, the minimum inactive period of three months might make sense from a software design perspective, but three month's delay in accessing vital information after someone unexpectedly dies could prove incredibly challenging in managing the estate of someone who has important financial, legal or other material locked away inside Google Drive, Gmail or any other Google service. Google's account support pages indicate that the Inactive Account Manager is the preferred means of dealing with a deceased user's account, but acknowledges that many people pass away without leaving clear instructions for their estate, nor activating Google's preferred tool (Google, 2017). If a proven immediate family member wants to get access to data in a deceased user's account, they have to provide evidence of their relationship with the deceased, scans of photographic identification, scans of the death certificate and other documentation. If this first process is successful, then Google will only commit to helping the user then file a court order issued in the United States before any actual access to the deceased user's data can be granted.

For the purposes of this chapter, what is most interesting is not the significant limitations of Google and Facebook's posthumous tools, but the presumptions underneath them. Both the Facebook Legacy Contact and Google Inactive Account Manager presume that social media users should be discussing the importance of their digital content and traces with other people. Both required users of nominate trusted family or friends who then explicitly share legal responsibility for that user's content. In effect, these tools algorithmically incorporate the idea of co-creation at their very core. They point to the importance of other users managing a user's social media legacy after they die, illustrating just how reliant our presences are on posthumous co-creators. Enshrining co-creative expectations in their deceased user tools, Facebook and Google thus explicitly recognize that social media accounts are not just about a relationship between the platforms and each individual user, but also about the shared trust between users who cocreate each other both in life and in death.

\section{The Algorithmic Afterlife}

While memorialization might sensibly appear the extent to which posthumous social media traces can circulate after a user's death, there are many companies and tools which seek to push the question of an algorithmic afterlife considerably further. A number of technology start-up companies have emerged which claim that their products will utilise digital traces and social media footprints to reanimate users in 
some fashion. The three most notable of these companies futuristic start-up companies - LivesOn (LivesOn, 2013), Eterni.me ("Eterni.me - Virtual Immortality," 2016) and Humai (Humai, 2016) - have all received significant media attention and made bold claims about the possibilities of digital tools to afford both forms of immortality and posthumous resurrection. Perhaps the most well-known of these three is LivesOn in part because of their memorable tagline, "When your heart stops beating, you'll keep tweeting". LivesOn invites users to allow proprietary algorithms to analyse their usual Twitter activity, 'learning' who they are through the way they tweet. A separate Twitter account (only visible to the user) starts to estimate what the 'real' account will say, with the expectation that the more accurate the service is, and the longer it runs, the closer the tweets will match. The eventual goal is that the digital version can take over, having 'learnt' the voice of that user, Tweeting in the user's name, long after they've passed away. Significantly, when the user dies, the digital version is placed in the hands of a nominated executor who, ultimately, decides the fate of this reanimated social media spectre. Eterni.me and Humai both make even broader claims about utilizing social media and digital tools further to facilitate an algorithmic afterlife, with the first purporting to facilitate posthumous chatbots with human-level intelligence and memories while the latter involves robotics and artificial intelligence in a more ambitious (or far-fetched) quest for a form of immortality. There are some dramatic presumptions underneath these tools, not least of which is the notion that the partial, context-specific traces left on social and digital media can ever be sufficient from which to fashion an even vaguely passable replica of a deceased person.

While algorithms can relatively easily estimate what advertising is most likely to be relevant (ie successful) given a sufficiently robust history of searches, choices or communication, Gillespie (2014) amongst others has argued that the it is the utilisation of algorithms as a predictive mechanism that raises at least as many, if not more, concerns. Extrapolating trends, likelihood and predictions from necessarily partial and often contextless data leads to all sorts of percentage-based presumptions which can reinforce cultural inequalities and normalise all sorts of discrimination justified by 'objective' algorithmic systems. Indeed, far from being in any way impartial, recent evidence has demonstrated that algorithms tend to replicate the cultural presumptions of their creators (Mager, 2012; Willson, 2017), often systematically and digitally reinforcing structural, cultural and historical forms of discrimination. Given the inequities and challenges in the very fibre of many contemporary algorithms, the notion that they might develop into systems that underpin some sort of posthumous reanimation based on the always incomplete traces left on social media is rife with problems that appear intractable from a contemporary perspective. If any form of algorithmic afterlife is possible, it seems more likely that these software entities will be something different and possibly unique, but never 'the same' as a deceased person.

These posthumous tools share with co-creation the recognition that all social media communication is always a form of media that can be drawn into other contexts and systems. While reanimation is stretching what these new contexts might be, the importance of social media users co-creating one another, and taking responsibility for posthumous management of digital traces, is made even more pressing in light of experiments with an algorithmic afterlife. Co-creating social media identities in this context may well involve deciding whether digital traces can be harnessed in ways which might just contradict the beliefs and wishes of the deceased user from which they originated. Co-creating death, just as co-creating new identities at birth, involves shared opportunities but also significant shared responsibilities, too. 


\section{Co-Created Conclusions}

Examining the way agency operates in terms of babies and the recently deceased reveals a great deal about our contemporary social media landscape, including limits on how useful it is to presume that all users and named individuals online will be able to actively shape and maintain their own web presences. The ways in which fetal ultrasound images are shared on Instagram reveals practices which confirm the importance of mothers and corporeality but simultaneously raise privacy concerns for babies who have not yet even been born. Infant wearables like the Owlet smart sock reveal ways in which infants are being captured as big data, and ask parents and others to carefully consider whether the reassurance these devices might provide justifies any potential privacy and rights issues later in life. As a marker of just how significant babies and infants are to big platforms and corporations, it's notable that in 2016 Google was awarded a patent for a 'smart crib' which includes a range of sensors and monitors that relay a range of information wirelessly and allow certain forms of manipulation of the crib, such as playing music or altering the surrounding temperature, via a smartphone app (US 2016/0183695, 2016). While this crib has not yet arrived on the market, the patent demonstrates that Google and other software giants see babies and infants as a growing area of interest. The management of recently deceased users' social media legacies, and their potential inclusion and manipulation in an algorithmic afterlife driven by tech start-up companies further reveals the importance of both having instructions in wills for digital estates, but also to the significant role loved ones will play in deciding on and shaping online legacies and memorials.

Situating social media use, big data generation and digital traces within the framework of co-creation strategically emphasizes with ways in which users are always contributing to the presence, shape and cohesion of each other's online presences. While collapsed contexts have been part of the initial definitional work on networked selves and networked publics (boyd, 2010), the liminal figures of the newly born and recently deceased sharply focus on exactly how broad those different contexts can be. Co-creation does not mean level terrain in terms of agency between users and platforms, nor does it mean a level terrain between users, as different users will have different levels and forms of agency across the lifespan, including prenatal traces and posthumous digital legacies. Co-creation as a lens makes explicit our roles in influencing the shape of each other online, and the power and responsibility we all have to exercise in doing so. Nowhere is this more evident than when we create content that names our children into being online, or when we must accept the role of shaping a recently deceased loved one's posthumous digital presence.

\section{References}

Acker, A., \& Brubaker, J. (2014). Death, Memorialization, and Social Media: A Platform Perspective for Personal Archives. Archivaria, 77, 2-23.

Aufderheide, P. (2010). Copyright, Fair Use, and Social Networks. In Z. Papacharissi (Ed.), A Networked Self: Identity, Community, and Culture on Social Network Sites (pp. 274-303). London \& New York: Routledge.

Bellamy, C., Arnold, M., Gibbs, M., Nansen, B., \& Kohn, T. (2013). Life beyond the timeline: creating and curating a digital legacy. Presented at the Prato CIRN Community Informatics Conference 2013, Prato, Italy. Retrieved from http://cirn.infotech.monash.edu/assets/docs/prato2013_papers/bellmay.pdf

Bonafide, C. P., Jamison, D. T., \& Foglia, E. E. (2017). The Emerging Market of Smartphone-Integrated Infant Physiologic Monitors. JAMA, 317(4), 353-354. https://doi.org/10.1001/jama.2016.19137 
boyd, danah. (2008). Facebook's Privacy Trainwreck: Exposure, Invasion, and Social Convergence. Convergence, 14(1), 13-20.

boyd, danah. (2010). Social Network Sites and Networked Publics: Affordances, Dymanics and Implications. In Z. Papacharissi (Ed.), A Networked Self: Identity, Community, and Culture on Social Network Sites (pp. 39-58). Routledge.

Brubaker, J. R., \& Callison-Burch, V. (2016). Legacy Contact: Designing and Implementing Post-mortem Stewardship at Facebook. In Proceedings of the 2016 CHI Conference on Human Factors in Computing Systems (pp. 2908-2919). New York, NY, USA: ACM. https://doi.org/10.1145/2858036.2858254

Burgess, J., \& Banks, J. (2010). User-Created Content and Online Social Networks. In S. Cunningham \& G. Turner (Eds.), The Media and Communications in Australia (3rd ed., pp. 295-306). Crows Nest, Australia: Allen \& Unwin.

Callison-Burch, V., Probst, J., \& Govea, M. (2015, February 12). Adding a Legacy Contact. Retrieved November 10, 2017, from https://newsroom.fb.com/news/2015/02/adding-a-legacy-contact/

Davis, J. L., \& Jurgenson, N. (2014). Context collapse: theorizing context collusions and collisions. Information, Communication \&amp; Society, 17(4), 476-485. https://doi.org/10.1080/1369118X.2014.888458

Eterni.me - Virtual Immortality. (2016). Retrieved November 14, 2014, from http://eterni.me/index.html

Gieseler, C. (2017). Gender-reveal parties: performing community identity in pink and blue. Journal of Gender Studies, 1-11. https://doi.org/10.1080/09589236.2017.1287066

Gillespie, T. (2014). The Relevance of Algorithms. In T. Gillespie, P. J. Boczkowski, \& K. A. Foot (Eds.), Media Technologies: Essays on Communication, Materiality, and Society (1 edition, pp. 167194). Cambridge, Massachusetts: The MIT Press.

Goffman, E. (1959). The Presentation of Self in Everyday Life. New York: Anchor Book.

Google. (2013). Inactive Account Manager. Retrieved November 10, 2017, from https://myaccount.google.com/inactive

Google. (2017). Submit a request regarding a deceased user's account. Retrieved November 10, 2017, from https://support.google.com/accounts/troubleshooter/6357590?hl=en\#ts=6357650

Heigl, K. (2017, March 22). Rest Assured Indeed! Retrieved August 21, 2017, from https://thoseheavenlydays.com/2017/03/22/rest-assured-indeed/

Humai. (2016). Humai - Transcending Human Biology Through Robotics. Retrieved July 12, 2016, from http://humaitech.com/

Hutchinson, J. (2017). Cultural Intermediaries: Audience Participation in Media Organisations. Cham, Switzerland: Palgrave Macmillan.

Jenkins, H. (2006). Convergence Culture: Where Old and New Media Collide (illustrated edition). NYU Press.

Johnson, S. A. (2014). "Maternal Devices", Social Media and the Self-Management of Pregnancy, Mothering and Child Health. Societies, 4(2), 330-350. https://doi.org/10.3390/soc4020330

King, D. (2014). Marketing wearable home baby monitors: real peace of mind? BMJ, 349, g6639. https://doi.org/10.1136/bmj.g6639

Lardinois, F. (2013, April 11). Google's New Inactive Account Manager Gives You Control Over Your Digital Afterlife. Retrieved November 10, 2017, from http://social.techcrunch.com/2013/04/11/googles-afterlife/

Leaver, T. (2013). The Social Media Contradiction: Data Mining and Digital Death. M/C Journal, 16(2). Retrieved from http://journal.mediaculture.org.au/index.php/mcjournal/article/viewArticle/625 
Leaver, T. (2015a). Born Digital? Presence, Privacy, and Intimate Surveillance. In Hartley, John \& W. Qu (Eds.), Re-Orientation: Translingual Transcultural Transmedia. Studies in narrative, language, identity, and knowledge (pp. 149-160). Shanghai: Fudan University Press.

Leaver, T. (2015b). Researching the Ends of Identity: Birth and Death on Social Media. Social Media + Society, 1(1). https://doi.org/10.1177/2056305115578877

Leaver, T. (2017). Intimate Surveillance: Normalizing Parental Monitoring and Mediation of Infants Online. Social Media + Society, 3(2). https://doi.org/10.1177/2056305117707192

Leaver, T., \& Highfield, T. (2018). Visualising the ends of identity: pre-birth and post-death on Instagram. Information, Communication \& Society, 21(1), 30-45. https://doi.org/10.1080/1369118X.2016.1259343

Leaver, T., \& Lloyd, C. (2015). Seeking Transparency in Locative Media. In R. Wilken \& G. Goggin (Eds.), Locative Media (pp. 162-174). London \& New York: Routledge.

LivesOn. (2013). LivesOn: Your Social Afterlife. Retrieved July 14, 2016, from http://liveson.org/connect.php

Livingstone, S., \& Third, A. (2017). Children and young people's rights in the digital age: An emerging agenda. New Media \& Society, 19(5), 657-670. https://doi.org/10.1177/1461444816686318

Lupton, D. (2013). The Social Worlds of the Unborn. Basingstoke: Palgrave MacMillan. https://doi.org/10.1057/9781137310729

Lupton, D., \& Thomas, G. M. (2015). Playing Pregnancy: The Ludification and Gamification of Expectant Motherhood in Smartphone Apps. M/C Journal, 18(5). Retrieved from http://journal.mediaculture.org.au/index.php/mcjournal/article/view/1012

Mager, A. (2012). Algorithmic Ideology. Information, Communication \& Society, 15(5), 769-787. https://doi.org/10.1080/1369118X.2012.676056

Marwick, A. E., \& boyd, danah. (2011). I tweet honestly, I tweet passionately: Twitter users, context collapse, and the imagined audience. New Media \& Society, 13(1), 114-133. https://doi.org/10.1177/1461444810365313

Marwick, A., \& Ellison, N. B. (2012). “There Isn't Wifi in Heaven!" Negotiating Visibility on Facebook Memorial Pages. Journal of Broadcasting \& Electronic Media, 56(3), 378-400. https://doi.org/10.1080/08838151.2012.705197

Owlet Baby Care. (2015). More Than Just A Gadget- The Owlet Vision. Retrieved from https://www.youtube.com/watch?v=fT9Vc68BfTI

Owlet Baby Care. (2017, June). Connected Care. Retrieved November 10, 2017, from http://www.owletcare.com/connected-care/

Papacharissi, Z. (2010). Conclusion: A Networked Self. In Z. Papacharissi (Ed.), A Networked Self: Identity, Community, and Culture on Social Network Sites (pp. 304-318). Routledge.

Pearson, E. (2009). All the World Wide Web's a stage: The performance of identity in online social networks. First Monday, 14(3). Retrieved from http://firstmonday.org/htbin/cgiwrap/bin/ojs/index.php/fm/article/view/2162/2127

Rettberg, J. W. (2014). Seeing Ourselves Through Technology: How We Use Selfies, Blogs and Wearable Devices to See and Shape Ourselves. New York: Palgrave MacMillan.

van der Nagel, E. (2017). From usernames to profiles: the development of pseudonymity in Internet communication. Internet Histories, 1(4), 312-331. https://doi.org/10.1080/24701475.2017.1389548

van Dijck, J. (2013). "You have one identity": performing the self on Facebook and Linkedln. Media, Culture \& Society, 35(2), 199-215. https://doi.org/10.1177/0163443712468605

van Dijck, J. (2014). Datafication, dataism and dataveillance: Big Data between scientific paradigm and ideology. Surveillance \& Society, 12(2), 197-208. 
van Dijck, J., \& Poell, T. (2016). Understanding the promises and premises of online health platforms. Big Data \& Society, 3(1), 1-11. https://doi.org/10.1177/2053951716654173

van Zoonen, L. (2013). From identity to identification: fixating the fragmented self. Media, Culture \& Society, 35(1), 44-51. https://doi.org/10.1177/0163443712464557

Willson, M. (2017). Algorithms (and the) everyday. Information, Communication \& Society, 20(1), 137150. https://doi.org/10.1080/1369118X.2016.1200645 